\title{
Demonstration of Active Guidance with SmartCane
}

\author{
Lawrence K. Au, Winston H. Wu, Maxim A. Batalin, Thanos Stathopoulos, William J. Kaiser \\ University of California, Los Angeles \\ ASCENT Lab, Electrical Engineering Department \\ au@ee.ucla.edu
}

\begin{abstract}
The usage of conventional assistive cane devices is critical in reducing the risk offalls, which are particularly detrimental for the elderly and disabled. Many of the individuals that experience the greatest risk of falling rely on cane devices for support of ambulation. However, the results of many studies have shown that incorrect cane usage is prevalent among cane users. The original SmartCane assistive system [4] has been developed to provide a method for acquiring detailed motion data from cane usage. The cane itself, however, lacks any type of programmability as well as real-time data processing algorithms to provide feedback to the cane user. In this demonstration, we have incorporated an embedded computing platform into SmartCane [2] and developed a real-time sensor information processing algorithm that provides direct detection of cane usage characteristics. The new system provides local data processing capability by classifying whether an individual is executing a stride with proper cane motion and applied forces. It also provides direct feedback information to the individual, thereby guiding the subject towards proper cane usage and reducing the risk of falls.
\end{abstract}

\section{Introduction}

Falls are particularly serious among the elderly and disabled where the number of individuals with fall-induced injury has been steadily increasing at a rate greater than accounted for in terms of demographic changes. Canes provide the required biomechanical support for mobility and are used by over four million individuals in the United States [3]. In many circumstances, however, the limitations of canes as assistive devices and the potential risk of falling resulting from cane usage can be ascribed to factors such as improper uses, abandoning of the cane (for reasons that may be the result of lack of training), disorders and disability resulting from repetitive stress, usage of the cane in the presence of environmental hazards including obstacles,

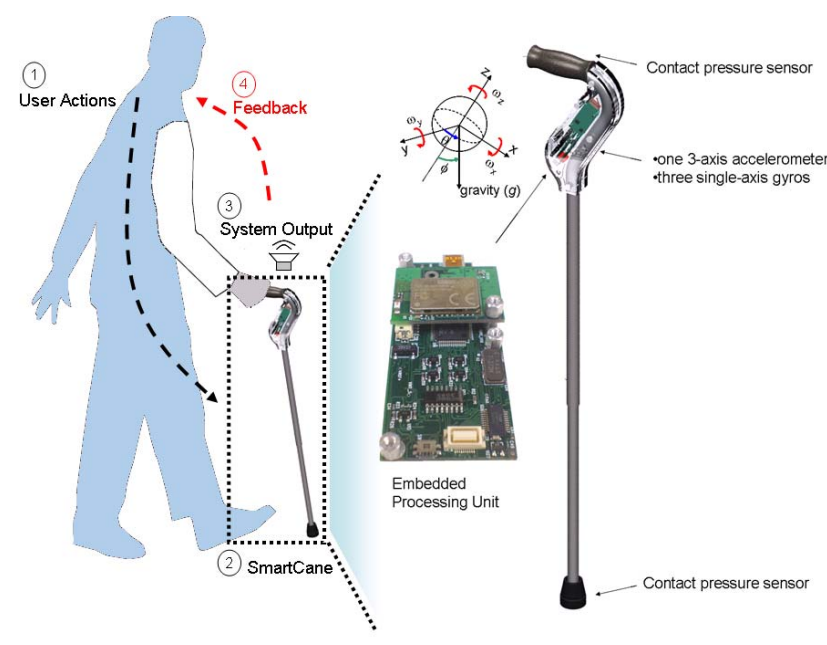

Figure 1. The SmartCane Architecture

stairs, and surfaces with uncertain support and friction [4].

Active guidance, therefore, becomes increasingly essential in training potential cane users. The original SmartCane system was developed to reduced the risks of falls associated with cane usage by providing direct sensor information regarding cane motion and applied forces during patient ambulation. To provide real-time feedback to the cane user and guide towards proper cane usage, however, processing algorithms must be integrated into SmartCane itself. This type of real-time classification provides the cane user an indication whether one has achieved proper cane strides. For medical professionals, the results from the classification may provide indications on whether a more individualized training is necessary for a particular cane user.

\section{System Implementation}

The SmartCane architecture comprises: (1) the cane user, (2) an embedded computing platform supporting sensor data acquisition, local data processing, Bluetooth wire- 


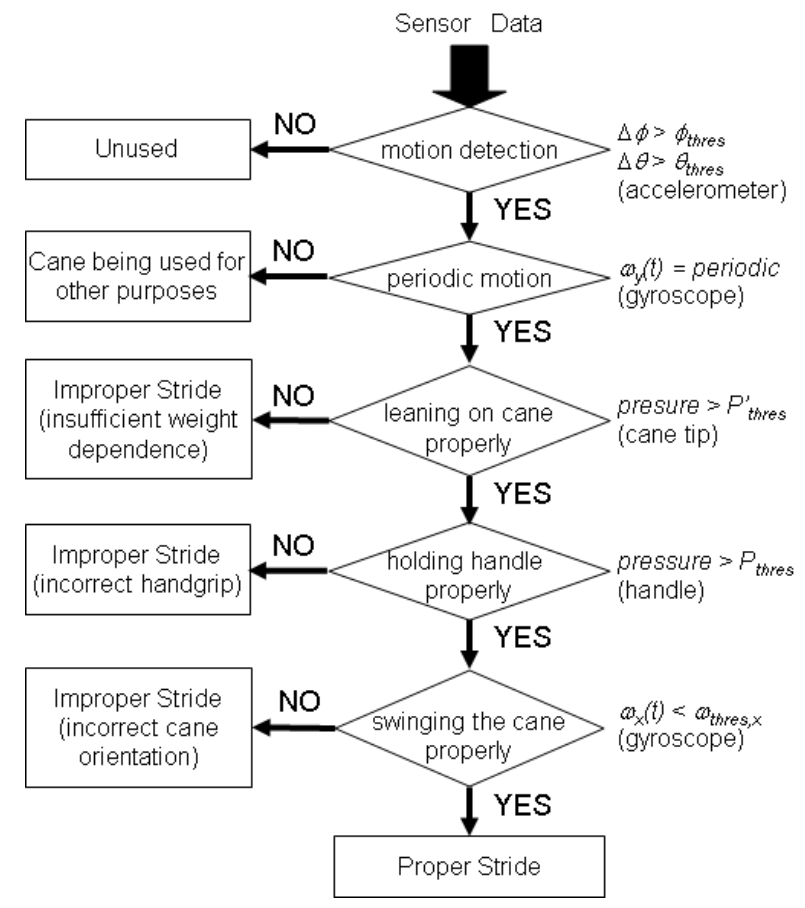

Figure 2. Classification Algorithm

\section{Data Analysis and Real-time Feedback}

One of the key objectives of the SmartCane system is to process real-time sensor data locally and provide appropriate feedback to the cane user regarding the state of the subject's cane usage, thereby guiding towards making proper strides. The main benefits associated with this approach include (1) immediate prevention of certain improper cane usage that can potentially lead to falls and various injuries, and (2) longer system lifetime (due to reduced energy consumption through local data processing).

The classification algorithm used on SmartCane is based on decision trees, as shown in Fig. 2. One main advantage of this approach is that the decision at each node can be made through threshold detections. Addtionally, cane users as well as medical professionals can interpret visually how SmartCane determines a proper stride. For instance, if the feedback of the SmartCane signals insufficient dependence on the cane, the cane user can immediately remedy such by placing more weight on the cane. Thresholds are determined through manual training. Based on these thresholds, the algorithm classifies, in real time, whether the subject is using the cane properly.

\section{Future Work}

We plan to expand the current architecture to include other wearable devices in order to provide a yet more detailed analysis of cane usage.

\section{References}

[1] L. Au, W. Wu, M. Batalin, D. McIntire, and W. Kaiser. Microleap: Energy-aware wireless sensor platform for biomedical sensing applications. IEEE BioCAS, Nov 27-30 2007.

[2] L. Au, W. Wu, M. Batalin, and W.J.Kaiser. Active guidance towards proper cane usage. 5th International Workshop on Wearable and Implantable Body Sensor Networks (BSN 2008), (Submitted).

[3] H. Bateni and B. E. Maki. Assistive devices for balance and mobility: benefits, demands, and adverse consequences. Arch Phys Med Rehabil, 86:134-145, 2005.

[4] W. Wu, L. Au, B. Jordan, T. Stathopoulos, M. Batalin, W. Kaiser, A. Vahdatpour, M. Sarrafzadeh, M. Fang, and J. Chodosh. The smartcane system: An assistive device for geriatrics. Third International Conference on Body Area Networks (BodyNets 2008), March 13-17, 2008.
The SmartCane uses a piezoelectric speaker to produce different audio tones, corresponding to different system states (and hence the state of the cane user). Cane users can adjust their movement based on the feedback, thereby self-guiding towards proper cane usage. 